\title{
Association of Contrast-Enhanced CT Findings with Etiology and Outcome in Patients with Portal Venous Gas and Pneumatosis Intestinalis: A Single Center Case Series Study
}

Naohiro Yoshida ( $\nabla$ yoshida_naohiro@med.kurume-u.ac.jp )

St Mary's Hospital https://orcid.org/0000-0002-0974-4263

Yoshihiko Sadakari

St Mary's Hospital

Kazuhito Tamehiro

St Mary's Hospital

Kazuma Ohkawara

St Mary's Hospital

Hiroyuki Nakane

St Mary's Hospital

Kayoko Endo

St Mary's Hospital

Kazuhisa Kaneshiro

St Mary's Hospital

Ayako Iwanaga

St Mary's Hospital

Munehiro Yoshitomi

St Mary's Hospital

Gentaro Hirokata

St Mary's Hospital

Takeshi Aoyagi

St Mary's Hospital

Toshiro Ogata

St Mary's Hospital

Masahiko Taniguchi

St Mary's Hospital

Research article 
Keywords: portal venous gas, pneumatosis intestinalis, contrast-enhanced CT findings, extra hepatic venous gas

Posted Date: June 7th, 2021

DOI: https://doi.org/10.21203/rs.3.rs-533515/v1

License: (c) (1) This work is licensed under a Creative Commons Attribution 4.0 International License. Read Full License 


\section{Abstract}

Background: Contrast-enhanced computed tomography (CT) is a reliable diagnostic tool often used to investigate the etiology of portal venous gas (PVG) and pneumatosis intestinalis (PI). However, there are few reports that offer an analysis of the relationship between particular CT findings and clinical outcomes. In this paper, we sought to close that gap and present the results of our analyses that reveal which CT findings are correlated with life-threatening cases.

Methods: 39 patients were diagnosed with PVG or PI from contrast-enhanced CT scans and underwent treatment at St. Mary's Hospital from January 2009 to December 2018. We reviewed patients' medical charts, laboratory data, and CT scan images retrospectively. We defined cases resulting in operation or death as group 1 , and patients with conservative treatment who survived as group 2 . We then analyzed the relationship between each CT finding and clinical feature.

Results: The primary underlying diseases identified for PVG and PI were bowel ischemia (13/39), enteritis (9/39), constipation (5/39), iatrogenic disease (4/39), and hemodialysis (3/39). Poor enhancement of the intestinal wall and extrahepatic venous gas were correlated with bowel ischemia $(p=0.0002$ and $p=0.0003$, respectively). Free air was less correlated with bowel ischemia $(p=0.02)$. Wall thickness was correlated with enteritis $(p=0.02)$. Mortality in cases with bowel ischemia and perforation was quite high $(60 \%)$ even if patients underwent surgery (33.3\%). Conversely, patients with enteritis, constipation, hemodialysis and gastric dilatation who did not require surgery resulted in more favorable outcomes with conservative treatment. In the multivariate analysis, extra hepatic venous gas was the only solitary factor correlated with group $1(p=0.0008)$.

Conclusions: Extra hepatic venous gas was the strongest predictive factor of clinical outcome in contrastenhanced CT findings correlated with bowel ischemia and required surgical treatment. The other CT findings were useful in diagnosing the underlying disease, but were not correlated with clinical outcomes. The necessity for surgical treatment remains dependent on the root cause.

\section{Background}

Portal venous gas (PVG) and pneumatosis intestinalis (PI) are often indicators of critical condition of intra-abdominal organs, such as bowel necrosis, and are associated with poor prognosis [1]. Recently, PVG and PI are identified more frequently due to the wide-spread use and improvement of computed tomography (CT) scans and ultrasonography (US) technology [2]. Early detection of PVG and PI can reveal the existence of non-lethal conditions that can be cured with conservative treatment [2-5].

PVG and PI are conditions in which air-shifts to mesenteric vessels or the intestinal wall from luminal organs such as the intestinal tract [6]. Major theories to explain the presence of PVG and PI are (1) airtranslocation through damaged intestinal mucosa, (2) elevated intraluminal pressure and (3) intramural gas-producing bacterial proliferation [7-9]. Various possible causes have been reported including bowel ischemia, gastrointestinal tract obstruction, gastrointestinal tract perforation, and gastrointestinal tract 
infection, although cholecystitis, pulmonary disease, digestive cancer, and decompression sickness have also been implicated $[1,4,10-15]$. Despite some progress, the definitive etiology of PVG and PI has not yet been elucidated, nor has it been determined what kinds of cases are life-threatening. Contrastenhanced CT scans are reliable examination tools for detecting PI and PVG, and these findings are often useful in diagnosing the underlying disease. In particular, contrast-enhanced CT findings can be helpful in predicting the prognosis of patients with PVG and PI. To date there are only a few reports that have analyzed particular CT findings related to $\mathrm{PI}$ and $\mathrm{PVG}$, and no reports have identified how these findings relate to prognosis [16-18].

Here we focused on contrast-enhanced CT findings accompanying PI and PVG. Then we analyzed the correlation between each CT finding and clinical outcome of PVG and PI to reveal which CT findings aid in diagnosis of the underlying disease, as well as predict which cases are life-threatening.

\section{Methods}

\section{Patients}

We obtained institutional review board approval from St. Mary's Hospital (Approval No: KEN19-0905). From January 2009 to December 2018, 39 patients were diagnosed with PVG or PI from contrastenhanced CT scans and underwent treatment at St Mary's Hospital. We reviewed patients' medical charts, laboratory data and CT scan images to gather information necessary for the case study.

\section{Contrast-enhanced CT Protocol}

We used multi-slice CT (2009-2016: Aquilion 64, 2017-2018: Aquilion PRIME, Canon Medical Systems Corporation, Japan) for diagnosis. We acquired 5-mm-thick contiguous slices when scanning patients, and after unenhanced CT scans were obtained, contrast-enhanced CT was performed. With the use of a power injector, $100 \mathrm{~mL}$ of nonionic contrast material (less than $60 \mathrm{~kg}: 300 \mathrm{mg} \mathrm{l} / \mathrm{mL}$, over $60 \mathrm{~kg}: 350 \mathrm{mg}$ $1 / \mathrm{mL}$ ) was injected intravenously at a rate of $3 \mathrm{~mL} / \mathrm{sec}$. Contrast-enhanced CT (Aquilion 64: 0.5-mm collimation, 0.828 pitch, $53 \mathrm{~mm} /$ rotation table speed, $0.5 \mathrm{sec}$ gantry rotation period; Aquilion PRIME: 0.5 $\mathrm{mm}$ collimation, 0.813 pitch, $65 \mathrm{~mm} /$ rotation table speed, $0.5 \mathrm{sec}$ gantry rotation period) was initiated 40 sec (early phase) and $180 \mathrm{sec}$ (late phase) after starting contrast material injection.

\section{Diagnosis of PVG, PI and Root Cause}

Gas within $2 \mathrm{~cm}$ of the liver border in CT scans was defined as PVG [19]. The presence of multiple gas foci within the intestinal wall was defined as PI [20]. In cases with no surgery, we diagnosed the underlying disease based on physical examinations, laboratory data and radiological diagnosis of the contrast-enhanced CT scan. In cases where surgery occurred, we diagnosed based on surgical findings 
and postoperative pathologic examination. Radiological diagnoses were performed by at least two inhospital radiologists.

\section{Assessments of Contrast-enhanced CT Findings}

We assessed the following six typical abdominal contrast-enhanced CT findings: intestinal dilatation, intestinal wall thickness, poor enhancement of intestinal wall, ascites, free air, and disproportionate fat stranding. We also assessed the existence of extra hepatic venous gas and the coexistence of PVG with PI.

We defined dilatation of intestines as a caliber greater than $2.5 \mathrm{~cm}$ for the small intestine and $4 \mathrm{~cm}$ for the colon [21]. We defined intestinal wall thickness as more than $3 \mathrm{~mm}$ in both the small intestine and colon [22].

\section{Patient Grouping for Analysis}

We divided cases into two groups based on outcomes. Cases that were suitable for operation were marked as group 1, and cases that were suitable for conservative treatment were placed in group 2 . We analyzed the differences of clinical features, contrast-enhanced CT findings and laboratory data between group 1 and group 2.

\section{Statistical Analysis}

Statistical analyses were performed with JMP® Pro 15. Data were expressed as the number of patients and ratio (\%) or mean \pm standard error (SE). The relationships between two factors were analyzed using the Mann-Whitney U test, chi-square test or Fisher's exact test. Univariate and multivariate analyses were performed using logistic regression, and a value of $P<0.05$ was considered significant.

\section{Results}

As shown in Table 1, 29 cases involved PVG, 6 cases involved PI, and 4 cases involved both phenomena. 17 patients were female and 22 were male. The mean age was $75.5 \pm 2.3$ (SE, 22-100). 16 patients had hypertension and 13 patients had diabetes mellitus, although other major pre-existing conditions such as heart failure and renal dysfunction were also identified.

The main causes of PVG and PI were bowel ischemia (13/39) and enteritis (9/39). Constipation (5/39), iatrogenic disease (4/39), hemodialysis (3/39) and gastrointestinal perforation (2/39) followed in frequency. Three of the iatrogenic cases were induced by the drug a-glucosidase inhibitor. One case was induced by ventilation during coma due to diabetic ketoacidosis. Other minor causes were bowel obstruction from adhesions (1/39), gastric dilatation (1/39), and cholecystitis (1/39). 
We then assessed the specific CT findings and analyzed their relationship to the diagnosis in order to reveal which CT findings can be useful in diagnosing the root problem (Table 2). Poor enhancement of the intestinal wall and extrahepatic venous gas were correlated with bowel ischemia $(p=0.0002$ and $p=$ 0.0003 , respectively). In PI cases, it was difficult to judge whether the intestinal wall was enhanced due to air trapped within. Free air was less correlated with bowel ischemia $(p=0.02)$. Wall thickness was correlated with enteritis $(p=0.02)$. Bowel obstruction, enteritis, hemodialysis, constipation, iatrogenic disease, gastric dilatation and cholecystitis were not correlated with any analyzed CT findings.

The flowchart of treatments and outcomes is shown in Fig. 1. We determined that 14 patients required surgery due to diagnoses such as suspected bowel necrosis, pan-peritonitis from gastrointestinal perforation, and cholecystitis. Of those, 7 patients underwent surgery (4 small bowel resections, 1 right colectomy, 1 cholecystectomy and 1 omental patch repair), while the remaining 7 patients refused due to generally poor health or advanced age. 25 patients who were diagnosed with enteritis, constipation, hemodialysis, simple bowel obstruction, gastric dilatation and iatrogenic disease underwent conservative treatments. In one case of bowel ischemia due to aortic dissection, we opted for conservative treatment as the ischemia was likely reversible. The patient recovered with fasting and fluid replacement. The mortality in bowel ischemia and perforation cases was quite high $(60 \%)$ even if the patients underwent surgery (33.3\%). Two other patients died despite undergoing surgery. One patient with perforated gastric cancer and multiple abdominal disseminations underwent omental patch repair of the hole, but later died from bleeding from the tumor on postoperative day 18. The other patient was diagnosed with widespread necrosis of the small intestine, and although we performed extensive intestinal resection, the patient died from septic shock on postoperative day 39. On the other hand, the patients with enteritis, constipation, hemodialysis and gastric dilatation who did not require surgery enjoyed more favorable outcomes with conservative treatment. Those outcomes suggest that understanding the disease underlying the PVG and $\mathrm{PI}$ is important because the diagnosis will determine whether an operation is necessary for treatment.

We analyzed the differences in clinical findings between groups 1 and 2 using univariate and multivariate analysis $($ Tables 3,4$)$. A broad comparison of all cases revealed that PVG was more closely correlated with group $1(p=0.04)$. In the univariate analysis, extrahepatic venous gas in contrast-enhanced CT findings $(p<0.0001)$, advanced age $(p=0.02)$, and a higher level of C-reactive protein (CRP) $(p=0.03)$ were correlated with group 1 . Interestingly, free air was correlated with group $2(p=0.04)$. The other $C T$ findings were not correlated with prognosis. In the multivariate analysis, extrahepatic venous gas was the only solitary factor correlated with group $1(p=0.0008)$.

\section{Discussion}

In this study, we analyzed the correlation between CT findings and clinical outcome of PVG and PI to reveal which CT findings can be useful in predicting life-threatening cases. We confirmed that the existence of extrahepatic venous gas was the strongest prognostic factor correlated with bowel ischemia. 
We also reinforced the best practice that treatment strategy be based on the unique characteristics of the underlying disease.

PVG was first described by Wolfe and Evans in 1955 in infants with necrotizing enterocolitis [23]. Liebman et. al. reported that the mortality rate was $75 \%$ and that surgical resection was considered the solitary life-saving treatment [7]. PI was first described in autopsy specimens by Du Vernoi in 1783 [20, 24]. Like PVG, PI was also implicated in mesenteric ischemia, and carried a high mortality rate of $33-44 \%$ $[6,24]$. As non-lethal cases of PVG are detected in recent years, the reported mortality of PVG is decreasing to $25-39 \%[3,4,7]$. Benign PI cases have also been reported in the literatures $[2,6,25]$.

Various diseases induce PVG and PI. The main causes of PVG and PI in our study were bowel ischemia, enteritis and constipation. We performed operations on the patients with bowel necrosis, gastrointestinal perforation, and cholecystitis, but the cases of bowel necrosis and perforation had a poor outcome. Koizumi et. al. reported in their large-scale data analysis that the major causes of PVG were bowel ischemia or necrosis $(53 \%)$, gastrointestinal obstruction or dilatation $(10.3 \%)$, and gastrointestinal infection (8.3\%) [10]. The mortality rates identified for bowel ischemia (26.8\%) and gastrointestinal perforation (33.3\%) were similar to our findings [10]. On the other hand, enteritis, constipation, hemodialysis, bowel obstruction without bowel ischemia, gastric dilatation, iatrogenic disease, and recoverable bowel ischemia were addressed with conservative treatment. This suggests that both PVG and $\mathrm{Pl}$ are not ominous signs by themselves, as there are non-fatal cases in which patients have recovered with conservative treatment $[11,26]$. Diverticulitis, inflammatory bowel disease, post-abdominal trauma, post-transplant surgery, barium enemas, and colonoscopy were also reported as non-lethal causes of PVG and PI $[11,25,27]$. The appropriateness of surgery as a treatment strategy can be determined by analyzing the underlying disease, as the most effective treatment strategies are directed at root cause rather than secondary manifestations $[4,5,11]$.

CT scans are highly-sensitive tools for detecting PVG and PI [3, 4, 7, 28]. Moreover, contrast-enhanced CT imaging is a useful investigative tool for identifying the underlying disease, especially bowel ischemia and gastrointestinal perforation [18, 28, 29]. We assessed representative CT findings of acute abdominal disease. Poor enhancement of the intestinal wall is a typical finding in bowel ischemia. Intestinal dilatation is the result of bowel obstruction, and sometimes accompanies aperistaltic bowel ischemia and enteritis. Intestinal wall thickness and disproportionate fat stranding are indicative of acute enteritis, inflammatory bowel disease, and gastrointestinal perforation [30, 31]. From the results of our study, we concluded that only the existence of extrahepatic venous gas was correlated with poor prognosis, while the other CT findings can aid in diagnosis of underlying disease. The detection of extrahepatic venous gas is relatively easy in a contrast-enhanced CT scan (Fig. 2). Some authors also reported that wide spread PVG was correlated with poor prognosis $[5,13,17,29]$. From a pathophysiological standpoint, wide spread PVG often indicates progressive mucosal damage and can reflect the severity of the patient's condition [32]. On the contrary, there are reports that the amount of gas was not related to patient prognosis at all $[12,33]$. Notably, the cases with free air in our study were correlated with better outcome, although free air is a finding often representative of high-mortality gastrointestinal perforation. Perhaps 
this is because $\mathrm{PI}$ is one of the causes for extraluminal free air, and some cases of PI accompanied free air without gastrointestinal perforation. In a previous study, it was reported that free air could occur with long-standing $\mathrm{PI}$ and is rarely associated with peritonitis [17]. The frequency of extraluminal free air with PI was reported at $24.7 \%$ [30]. The combination of PVG and PI is not related to outcome in our study, although some authors reported that it related to worse outcome $[6,25]$. Overall, we suggest that extra hepatic venous gas and poor enhancement of the intestinal wall are useful findings to predict bowel ischemia as well as determine the suitability of surgical treatment. Special caution should be used in cases with free air and PI in order to avoid futile laparotomy.

In addition to CT scan findings, data garnered from physical examinations, vital signs and laboratory tests were also prognostic factors for PVG and PI in previous literature. Higashizono et. al. reported that elevated levels of CRP, creatinine and lactate were correlated with bowel necrosis [1]. Abboud et. al. suggested that pre-existing chronic illnesses such as chronic renal failure, diabetes mellitus and hypertension affect the outcomes [11]. But the factors reported were numerous, and there is no consensus on which factor is the most reliable. In our study, advanced age and elevated CRP were significantly associated with severe cases in the univariate analysis.

$P V G$ is considered to be an exacerbated form of PI with the same pathophysiology $[2,24,34]$. Since the liver is the drainage site in PI, PVG may indeed signal an advanced stage of PI [2]. Actually, PVG cases were related with group 1 more than with PI in this study. However, we should keep in mind that some cases of PI may include severe cases.

Our study was limited by a few factors: exclusion of data from institutions other than our own, relatively small sample size, and heterogeneity of patient conditions at first examination, as patients presented with variable physical manifestations after transfer to our hospital. There was also a degree of unfavorable selection bias of cases in regard to surgical treatment: some patients who required surgery could not receive the appropriate treatment due to their physical condition or advanced age.

\section{Conclusions}

In our study, the existence of extrahepatic venous gas was the strongest predictive factor for clinical outcome in contrast-enhanced CT findings. The other CT findings, although not correlated with clinical outcome, were useful in diagnosing the underlying diseases. This underscores the importance of obtaining high-quality contrast-enhanced CT images when appropriate, as determining the necessity for surgery is highly dependent on understanding the nature of the underlying disease.

\section{Abbreviations}

PVG: Portal venous gas; PIP: pneumatosis intesnalis; CT: Computed tomography; US: Ultrasonography; SE: Standard error; CRP: C-creative protein 


\section{Declarations}

Acknowledgements

We would like to thank Brandi Jones for English language editing.

\section{Author's contributions}

NY designed the study concept, analyzed the data, and wrote the manuscript. YS, TO, and MT contributed to the revision of the manuscript for important intellectual content. $\mathrm{KT}, \mathrm{KO}, \mathrm{HN}, \mathrm{KE}, \mathrm{KK}, \mathrm{Al}, \mathrm{MY}, \mathrm{GH}$, and TA collected the patient's clinical data. All authors read and approved the final manuscript.

\section{Funding}

Not applicable.

\section{Availability of data and materials}

The datasets used or analyzed during the current study are available from the corresponding author on reasonable request.

\section{Ethics approval and consent to participate}

This study was approved by the Institutional Review Board of St. Mary's Hospital, Fukuoka (Approval No: KEN19-0905).

\section{Consent for publication}

All patients gave consent for publication.

\section{Competing interests}

The authors declare that they have no competing interests.

\section{References}

1. Higashizono K, Yano H, Miyake O, Yamasawa K, Hashimoto M. Postoperative pneumatosis intestinalis (PI) and portal venous gas (PVG) may indicate bowel necrosis: a 52-case study. BMC Surg. 2016;16(1):42.

2. Wayne E, Ough M, Wu A, Liao J, Andresen KJ, Kuehn D, Wilkinson N. Management algorithm for pneumatosis intestinalis and portal venous gas: treatment and outcome of 88 consecutive cases. $J$ Gastrointest Surg. 2010;14(3):437-48.

3. Faberman RS, Mayo-Smith WW. Outcome of 17 patients with portal venous gas detected by CT. AJR Am J Roentgenol. 1997;169:1535-8. 
4. Kinoshita H, Shinozaki M, Tanimura H, Umemoto Y, Sakaguchi S, Takifuji K. et.

5. al. Clinical features and management of hepatic portal venous gas: four case.

6. reports and cumulative review of the literature. Arch Surg. 2001;136:1410-4.

7. Hussain A, Mahmood H, El-Hasani S. Portal vein gas in emergency surgery. World J Emerg Surg. 2008;3:21.

8. Morris MS, Gee AC, Cho SD, Limbaugh K, Underwood S, Ham B, Schreiber MA. Management and outcome of pneumatosis intestinalis. Am J Surg. 2008;195(5):679-82. discussion 682 - 673.

9. Liebman PR, Patten MT, Manny J, Benfield JR, Hechtman HB. Hepatic- Portal Venous Gas in Adults: Etiology, Pathophysiology and Clinical Significance. Ann Surg. 1978;187:281-7.

10. Nelson AL, Millington TM, Sahani D, Chung RT, Bauer C, Hertl M, et al. Hepatic portal venous gas: the ABCs of management. Arch Surg. 2009;144:575-81. discussion 81.

11. Gonda M, Osuga T, Ikura Y, Hasegawa K, Kawasaki K, Nakashima T. Optimal treatment strategies for hepatic portal venous gas: Aretrospective assessment. World J Gastroenterol. 2020;26(14):1628-37.

12. Koizumi C, Michihata N, Matsui H, Fushimi K, Yasunaga H. In-Hospital Mortality for Hepatic Portal Venous Gas: Analysis of 1590 Patients Using a Japanese National Inpatient Database. World J Surg. 2018;42(3):816-22.

13. Abboud B, El Hachem J, Yazbeck T, Doumit C. Hepatic portal venous gas: physiopathology, etiology, prognosis and treatment. World J Gastroenterol. 2009;15(29):3585-90.

14. Alqahtani S, Coffin CS, Burak K, Chen F, MacGregor J, Beck P. Hepatic portal venous gas: a report of two cases and a review of the epidemiology, pathogenesis, diagnosis and approach to management. Can J Gastroenterol. 2007;21(5):309-13. doi:10.1155/2007/934908.

15. Li Z, Su Y, Wang X, Yan H, Sun M, Shu Z. Hepatic portal venous gas associated with colon cancer: $A$ case report and literature review. Med (Baltim). 2017;96(50):e9352.

16. Napolitano L, Waku M, Costantini R, Mazahreh T, Innocenti P. Portal vein gas due to gangrenous cholecystitis treated by a laparoscopic procedure: report of a case. Surg Today. 2009;39(10):909-12.

17. Morita S, Yamagiwa T, Inokuchi S. Portal venous gas on computed tomography imaging in patients with decompression sickness. J Emerg Med. 2013;45(1):e7-11.

18. Bani Hani M, Kamangar F, Goldberg S, Greenspon J, Shah P, Volpe C, Turner DJ, Horton K, Fishman EK, Francis IR, et al. Pneumatosis and portal venous gas: do CT findings reassure? J Surg Res. 2013;185(2):581-6.

19. Ko S, Hong SS, Hwang J, Kim HJ, Chang YW, Lee E: Benign versus life-threatening causes of pneumatosis intestinalis: differentiating CT features. Rev Assoc Med Bras (1992) 2018, 64(6): 543548.

20. Koami H, Isa T, Ishimine T, Kameyama S, Matsumura T, Yamada KC, Sakamoto Y. Risk factors for bowel necrosis in patients with hepatic portal venous gas. Surg Today. 2015;45(2):156-61.

21. Elsayes KM, Shaaban AM, Rothan SM, Favadi S, Madrazo BL, Castillo RP, et al. A Comprehensive Approach to Hepatic Vascular Disease. Radiogrphics. 2017;37(3):813-36. 
doi:10.1148/rg.2017160161.

22. Takami Y, Koh T, Nishio M, Nakagawa N. Pneumatosis intestinalis leading to perioperative hypovolemic shock: Case report. World J Emer Surg. 2011;6:15.

23. Furukawa A, Yamasaki M, Furuichi K, Yokoyama K, Nagata T, Takahashi M, et al. Helical CT in the diagnosis of small bowel obstruction. Radiogrphics. 2001;21(2):341-55. doi:10.1148/radiographics.21.2.g01mr05341.

24. Naguib N, Mekhail P, Gupta V, Naguib N, Masoud A. Portal venous gas and pneumatosis intestinalis; radiologic signs with wide range of significance in surgery. J Surg Educ. 2012;69(1):47-51.

25. Wolfe JN, Evans WA. Gas in the portal veins of the liver in infants; a roentgenographic demonstration with postmortem anatomical correlation. Am J Roentgenol Radium Ther Nucl Med. 1955;74(3):4868.

26. JG DV. Anatomische Beobachtungen der Unter der Aussern und Innern Haut der Gedarme Eingeschlossenen Luft. Phys Med Abhndl Acad Wissenschin Petersb 1783, 2: 182.

27. Mallappa S, Warren OJ, Kantor R, Mohsen Y, Harris S. Pneumatosis intestinalis and hepatic portal venous gas on computed tomography - a non-lethal outcome. JRSM Short Rep. 2011;2(11):88.

28. Monneuse O, Pilleul F, Barth X, Gruner L, Allaouchiche B, Valette PJ, Tissot E. Portal venous gas detected on computed tomography in emergency situations: surgery is still necessary. World J Surg. 2007;31(5):1065-71.

29. Taniguchi K, Asakuma M, Nagayabu K, Takashima S, lida R, Hirokawa F, Umegaki O, Neo M, Takasu A, Uchiyama K. Exploring the use of single-port surgery in the conservative management of hepatic portal vein gas: A case report. Med (Baltim). 2018;97(47):e13368.

30. Schindera ST, Triller J, Vock P, Hoppe H. Detection of hepatic portal venous gas: its clinical impact and outcome. Emerg Radiol. 2006;12(4):164-70.

31. Moussa M, Marzouk I, Abdelmoula K, Manamani A, Dali N, Farhat LC, Hendaoui L. Role of Computed tomography in predicting prognosis of Hepatic portal venous gas. Int J Surg Case Rep. 2017;30:177-82.

32. Adachi W, Matsushita T, Yashiro Y, Imura J, Shiozawa H, Kishimoto K. Clinical characteristics of pneumoperitoneum with pneumatosis intestinalis detected using computed tomography: A descriptive study. Med (Baltim). 2020;99(40):e22461.

33. Allgayer $\mathrm{H}$, Braden B, Dietruch CF. Transabdominal ultrasound in inflammatory bowel disease. Conventional and recently developed techniques - Update. Medical Ultrasonography. 2011;13(4):302-13.

34. Heye T, Bernhard M, Mehrabi A, Kauczor HU, Hosch W. Portomesenteric venous gas: is gas distribution linked to etiology and outcome? Eur J Radiol. 2012;81(12):3862-9.

35. Martínez J-L, González-Acosta J. Portal venous gas. Report of three cases. Ann Hepatol. 2009;8(2):151-5. 
36. Susman NSH. Gas embolization of the portal venous system. Am J Roentgenol Radium Ther Nucl Med. 1960;83:847-50.

\section{Tables}

Table 1. Patient characteristics, pre-existing disease, and underlying disease.

\begin{tabular}{ll}
\hline & $\begin{array}{l}\text { Total } \\
n=39(\%)\end{array}$ \\
\hline PVG & $29(74.4 \%)$ \\
PI & $6(15.4 \%)$ \\
PVG+PI & $4(10.3 \%)$ \\
Age (mean \pm SE) & $75.5 \pm 2.3$ \\
Gender & \\
Female & $17(43.6 \%)$ \\
Male & $22(56.4 \%)$ \\
Underlying disease & \\
Hypertension & $16(41 \%)$ \\
Diabetes melitus & $13(33.3 \%)$ \\
Renal disease & $7(17.9 \%)$ \\
Heart Failure & $4(10.3 \%)$ \\
Hemodialysis & $3(7.7 \%)$ \\
Diagnosis & \\
Bowel ischemia & $13(33.3 \%)$ \\
Enteritis & $9(23.1 \%)$ \\
Constipation & $5(12.8 \%)$ \\
latrogenic & $4(10.3 \%)$ \\
Hemodialysis & $3(7.7 \%)$ \\
Gastrointestinal tract perforation & $2(5.1 \%)$ \\
Bowel obstruction & $1(2.6 \%)$ \\
Gastric dilatation & $1(2.6 \%)$ \\
Cholecystitis & $1(2.6 \%)$ \\
\hline
\end{tabular}

Table 2. The relationships between particular CT findings and underlying disease.

$<p>$ Due to technical limitations, table 2 is only available as a download in the Supplemental Files section. $</ p>$

Table 3. Comparison of clinical features between two groups (univariate analysis). 


\begin{tabular}{|c|c|c|c|c|c|}
\hline & & $\begin{array}{l}\text { Group1 } \\
(n=15)\end{array}$ & $\begin{array}{l}\text { Group2 } \\
(n=24)\end{array}$ & $\mathrm{p}$ value & odds ratio \\
\hline PVG: PI & & $15: 0$ & $18: 6$ & 0.04 & NA \\
\hline \multirow[t]{8}{*}{ CT findings } & Intestinal dilatation & 8 & 9 & 0.33 & 1.9 \\
\hline & Intestinal wall thickness & 7 & 14 & 0.48 & 0.6 \\
\hline & Low enhancement of intestinal wall & 6 & 6 & 0.33 & 2 \\
\hline & Ascites & 7 & 12 & 0.84 & 0.9 \\
\hline & Free air & 1 & 8 & 0.04 & 0.1 \\
\hline & Disproportionate fat stranding & 4 & 7 & 0.87 & 0.88 \\
\hline & Extra hepatic venous gas & 9 & 1 & $<0.0001$ & 34.5 \\
\hline & Combination of PVG and PI & 0 & 4 & 0.1 & $\min$ \\
\hline \multicolumn{2}{|c|}{ Age (mean $\pm \mathrm{SE}$ ) } & $70.2 \pm 3.3$ & $82.6 \pm 2.3$ & 0.02 & 1.1 \\
\hline Gender & Female: Male & 8: 7 & $9: 15$ & 0.33 & 0.5 \\
\hline \multirow[t]{9}{*}{ Laboratory data } & $\mathrm{sBP}(\mathrm{mmHg})$ & $110 \pm 9$ & $116 \pm 6$ & 0.59 & 0.99 \\
\hline & $\operatorname{WBC}(/ \mu \mathrm{L})$ & $11963 \pm 1770$ & $10731 \pm 1400$ & 0.59 & 1 \\
\hline & $\mathrm{Hb}(\mathrm{g} / \mathrm{dL})$ & $11.1 \pm 0.5$ & $11.8 \pm 0.4$ & 0.28 & 0.83 \\
\hline & $\mathrm{CRP}(\mathrm{mg} / \mathrm{dL})$ & $7.1 \pm 1.5$ & $2.9 \pm 1.2$ & 0.04 & 1.13 \\
\hline & $\mathrm{Alb}(\mathrm{g} / \mathrm{dL})$ & $3 \pm 0.2$ & $3.2 \pm 0.2$ & 0.5 & 0.75 \\
\hline & $\mathrm{Lac}(\mathrm{mg} / \mathrm{dL})$ & $12.2 \pm 6.8$ & $3.8 \pm 7.9$ & 0.46 & 1.1 \\
\hline & $\operatorname{BUN}(\mathrm{mg} / \mathrm{dL})$ & $30 \pm 5$ & $36 \pm 5$ & 0.42 & 0.99 \\
\hline & AST (IU/L) & $150 \pm 48$ & $51 \pm 38$ & 0.11 & 1 \\
\hline & Cre $(\mathrm{mg} / \mathrm{dL})$ & $1.5 \pm 0.4$ & $2.2 \pm 0.3$ & 0.19 & 0.74 \\
\hline \multirow[t]{5}{*}{ Underlying disease } & Hypertension & 6 & 10 & 0.92 & 0.93 \\
\hline & Diabetes melitus & 4 & 9 & 0.48 & 0.61 \\
\hline & Renal disease & 2 & 5 & 0.55 & 0.58 \\
\hline & Heart Failure & 3 & 1 & 0.12 & 5.8 \\
\hline & Hemodialysis & 0 & 3 & 0.08 & $\min$ \\
\hline
\end{tabular}

BP blood pressure, WBC white blood cell, BUN blood urea nitrogen, AST aspartate aminotransferase

Table 4. Comparison of clinical features between two groups (multivariate analysis).

\begin{tabular}{llll}
\hline & $95 \% \mathrm{CI}$ & p value & odds ratio \\
\hline Free air & $0.21-79$ & 0.15 & 0.24 \\
Extra hepatic venous gas & $2.41-329$ & 0.0008 & 28.1 \\
Age & $0.86-1.03$ & 0.15 & 1.06 \\
CRP & $0.85-1.12$ & 0.68 & 1.03 \\
\hline
\end{tabular}

CI confidence interval

\section{Figures}




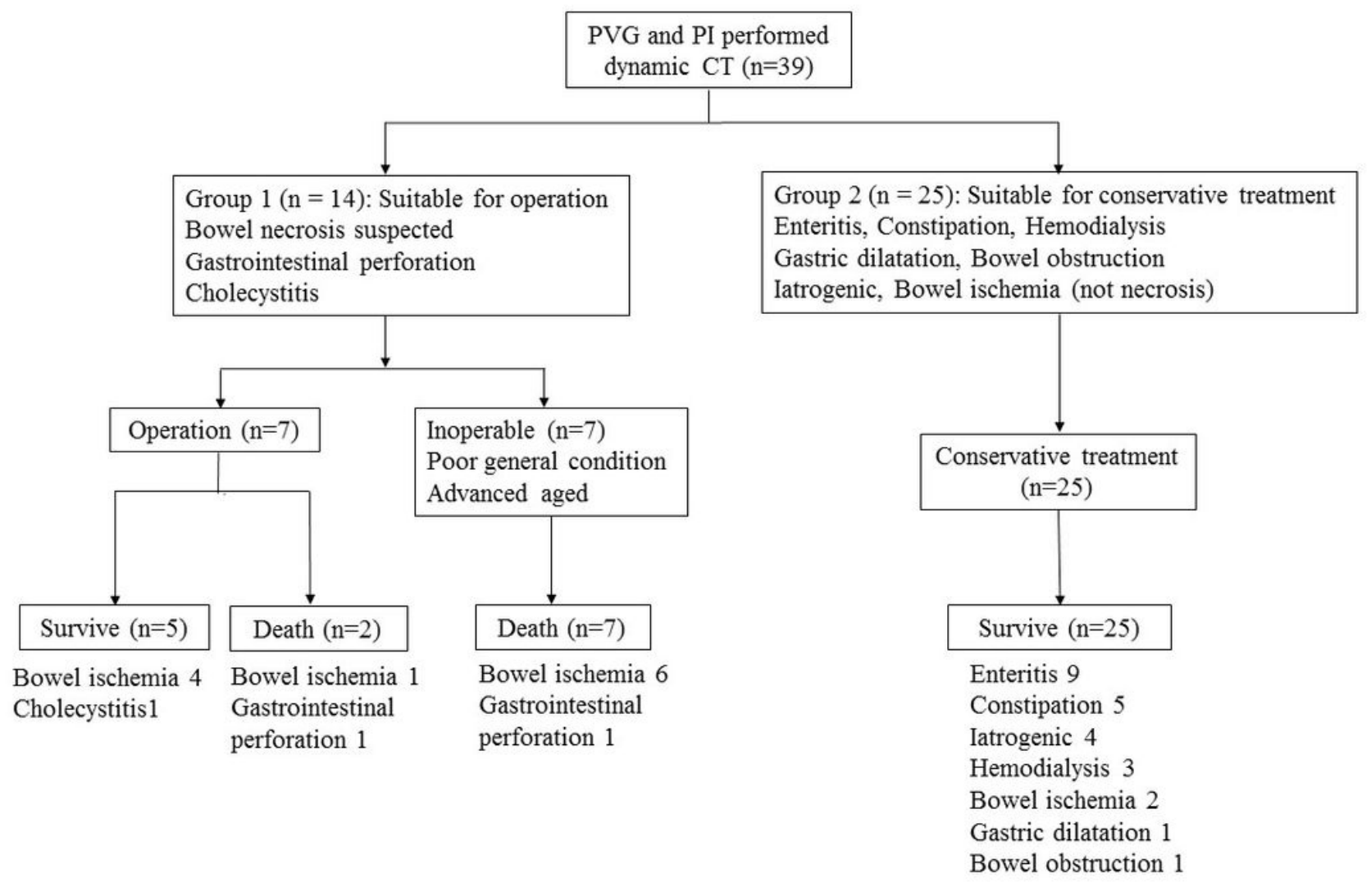

\section{Figure 1}

Flowchart of treatments and outcomes.
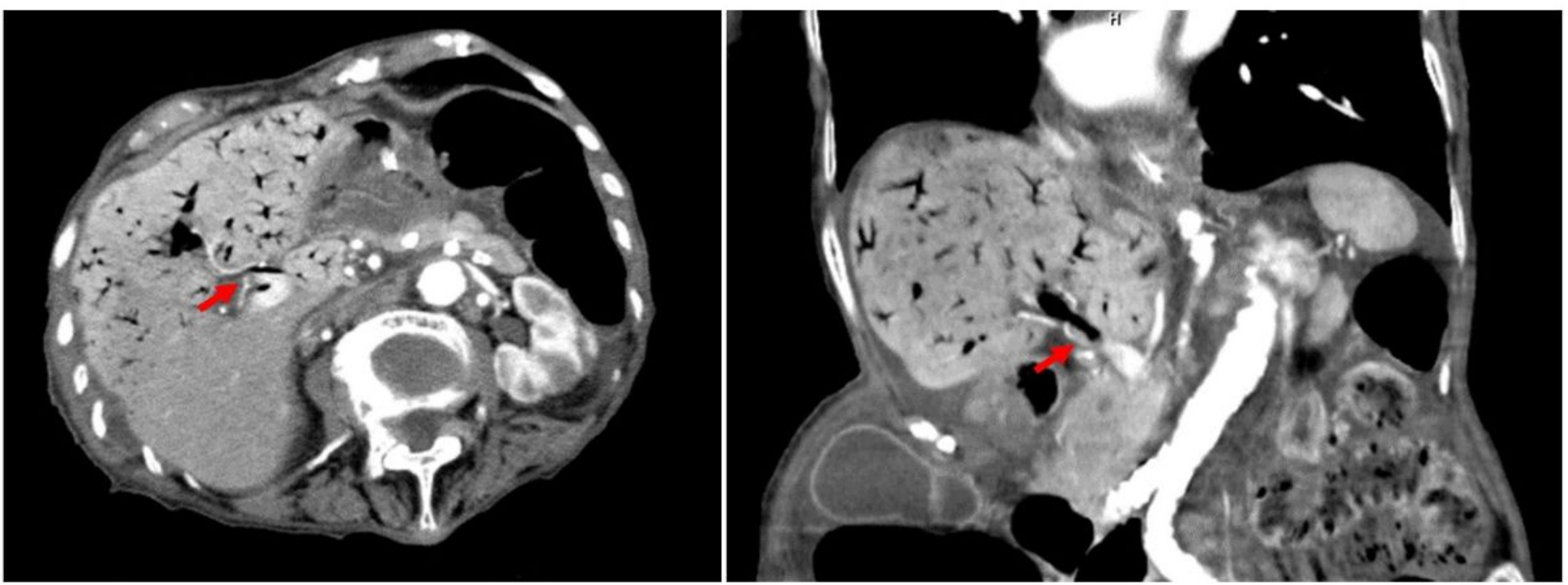
Figure 2

The image of extra hepatic venous gas in contrast-enhanced CT scan (arrow).

\section{Supplementary Files}

This is a list of supplementary files associated with this preprint. Click to download.

- PVGPITable2.xIsx 